\title{
A Dioid Linear Algebra Approach to Study a Class of Continuous Petri Nets
}

\author{
Duan Zhang, Huaping Dai, Youxian Sun \\ National Laboratory of Industrial Control Technology, Zhejiang University, Hangzhou, \\ P.R.China, 310027 \\ \{dzhang, hpdai, yxsun\}@iipc.zju.edu.cn
}

\begin{abstract}
Continuous Event Graphs (CEGs), a subclass of Continuous Petri Nets, are defined as the limiting cases of timed event graphs and Timed Event Multigraphs. A set of dioid algebraic linear equations will be inferred as a novel method of analyzing a special class of CEG, if treated the cumulated token consumed by transitions as state-variables, endowed the monotone nondecreasing functions pointwise minimum as addition, and endowed the lower-semicontinuous mappings, from the collection of monotone nondecreasing functions to itself, the pointwise minimum as addition and composition of mappings as multiplication. As a new modeling approach, it clearly illustrate characteristic of continuous events. Based on the algebraic model, an example of optimal Control is demonstrated.
\end{abstract}

\section{Introduction}

It is well know that max-plus algebra is powerful tools for Discrete Event Dynamic Systems (DEDS) [1-3]. Min-plus algebra is the dual of max-plus algebra, and they are both dioid, an idempotent semiring.

Linear model is so popular that it is adopted by most of classic control theory. Many evidences support that max-plus algebra, min-plus algebra, dioid and some other idempotent semirings are appropriate mathematic tools to describe the phenomena of DEDSs, especially synchronization. With the operations of above algebraic systems, some logic nonlinear formulae come to linear formulae. As a byproduct of the linearization, the existence of an eventual periodic regime is readily obtained, the performance being characterized in terms of invariants of the original net [3].

An event graph is a Petri net such that each place has only one input arc and one output arc. Timed Event Graphs (TEG) are a subclass of event graphs satisfied that the tokens have to stay a minimum amount of time in the places. The dynamic of TEGs can be represented as a max-plus linear algebraic model [1]. Timed Event Multigraphs (TEMGs), each arc has an integral weight, are extensions of TEGs. The max-plus linear algebraic model of TEMGs was built by G. Cohen [2] and HuaPing Dai [3] in different way respectively. By those linear formulae, the analyses of TEGs and TEMGs are analogous to traditional linear system theory. 
Comparing with TEGs or TEMGs, Continuous Event Graphs (CEGs), proposed by R. David and H. Alla [4-6], have continuous places and continuous transitions. As a limiting case of TEMGs, CEGs are applied to describe the continuous events system [7-11], for example the flow control and congest control of TCP [12]. Unfortunately, the building of algebraic model for CEGs is more difficult than TEMGs. G. Cohen [2] induce a min-plus algebraic model considered only the case of continuous places, but no the case of continuous transitions. There is no universal algebraic model is constructed for CEGs so far.

In Section 2, the definition of CEG is reviewed. Some properties of CEGs will be presented in Section 3. A dioid linear algebraic representation of a class of CEGs is given in Section 4 and an example will tackled in Section 5 with the new model.

\section{Definition of CEG}

Let $\mathrm{R}$ is the set of real, $\varpi=+\infty, \overline{\mathrm{R}}=\mathrm{R} \bigcup\{\varpi\}$ and $\mathrm{R}^{+}=[0,+\infty) \bigcup\{\varpi\}$.

Definition 1. A CEG is a 6-tuplet: $<P, T, R, W, V, M_{0}>; P$ is a set of continuous places; $T$ is a set of continuous transitions; $R=R_{P T} \cup R_{T P}$, where $R_{P T} \subseteq P \times T$ and $R_{T P} \subseteq T \times P,<n_{1}, n_{2}>\in R$ represent that $n_{1}$ is a input of $n_{2}$ and $n_{2}$ is a output of $n_{1} ; W: R \rightarrow \mathrm{R}^{+}$is the weight of $R ; V: T \rightarrow \mathrm{R}^{+}$is the maximum firing velocities of transitions; $M_{0}: P \rightarrow \mathrm{R}^{+}$is the initial markings of places; every place has only one input transition and one output transition; moreover we assume that every transition have at least one input place and one output place.

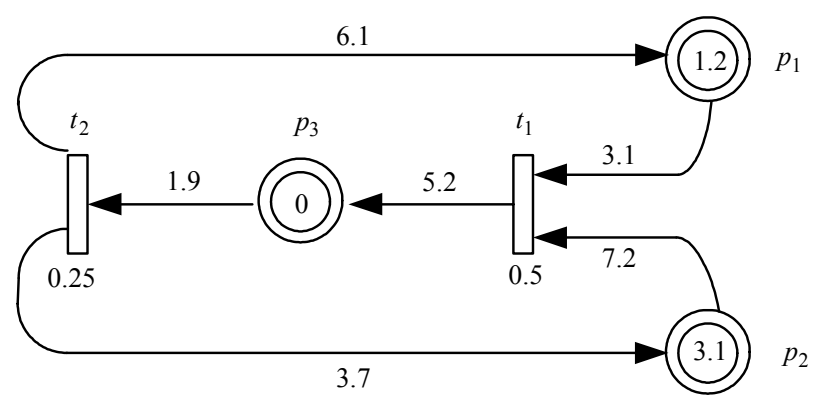

Fig. 1. A CEG

CEGs can be illustrated by directed graphs (Fig. 1) that denote continuous places as double circle, denote continuous transitions as rectangle, and denote the elements of $R$ as arrow.

Definition 2. Let $t$ be a transition, ${ }^{\circ} t=\left\{p \in P \mid<p, t>\in R_{P T}\right\}$ is called the preset of $t, t^{\circ}=\left\{p \quad \in P \mid<t, p>\in R_{T P}\right\}$ is called the postset of $t$; Let $p$ be a place, 
${ }^{\circ} p=\left\{t \in T \mid<t, p>\in R_{T P}\right\}$ is called the preset of $p, \quad p^{\circ}=\left\{t \in T \mid<p, t>\in R_{P T}\right\}$ is called the postset of $p$.

Definition 3. Let $\operatorname{Mark}(p, \tau)$ denote the token of place $p$ at the epoch $\tau, \bar{V}(t, \tau)$ denote the firing velocity of transition $t$, then:

1) $\bar{V}(t, 0)=0$;

2) if $\forall p \in{ }^{\circ} t \operatorname{Mark}(p, \tau)>0$, then $\bar{V}(t, \tau)=V(t)$;

3) if $\exists p \in{ }^{\circ} t \operatorname{Mark}(p, \tau)=0$, denote $T_{0}=\left\{t_{0} \in{ }^{\circ} p \mid p \in{ }^{\circ} t\right.$ and $\left.\operatorname{Mark}(p, \tau)=0\right\}$, then $\bar{V}(t, \tau)=\min \left\{\min _{p \in T_{0}}\left\{\bar{V}\left({ }^{\circ} p, \tau\right) \times W\left({ }^{\circ} p, p\right) / W(p, t)\right\}, V(t)\right\}$. Note that if there is a loop satisfied that for any place $p$ in the $\operatorname{loop} \operatorname{Mark}(p, \tau)=0$, then for any transition $t$ in the loop, $\bar{V}(t, \tau)=0$.

Remark 1. The firing of transition $t$ can lead change of the token of some places:

1) if $p \in t^{\circ}$, then $\int_{\tau_{2}}^{\tau_{1}} W(t, p) \bar{V}(t, \tau) \cdot d \tau$ represents the mark input $p$ from $t$ in time interval $\left[\tau_{1}, \tau_{2}\right]$;

2) if $p \in{ }^{\circ} t$, then $\int_{\tau_{2}}^{\tau_{1}}-W(p, t) \bar{V}(t, \tau) \cdot d \tau$ represents the mark input $t$ from $p$ in time interval $\left[\tau_{1}, \tau_{2}\right]$.

\section{Properties of CEGs}

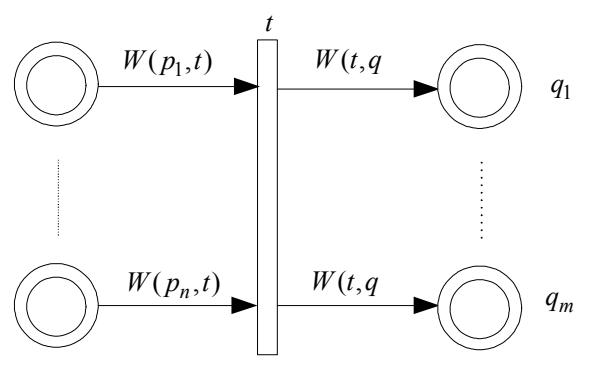

Fig. 2. A continuous transition

Definition 4. $M_{(\cdot)}:[0,+\infty) \rightarrow \mathrm{R}^{+}$is a monotone nondecreasing function:

1) if $t \in T, M_{t}(\tau)$ is the total mark $t$ consumed from the epoch 0 to $\tau$, namely $M_{t}(\tau)=\int_{0}^{\tau} \bar{V}(t, u) d u$ 
2) if $p \in P, M_{p}(\tau)$ is the total mark entered $p$ from the epoch 0 to $\tau$, namely $M_{p}(\tau)=M_{0}(p)+\int_{0}^{\tau} \bar{V}(t, u) d u ;$

Then we consider transition $t$ with $^{\circ} t=\left\{p_{1}, p_{2}, \ldots, p_{n}\right\}$ and $t^{\circ}=\left\{q_{1}, q_{2}, \ldots, q_{m}\right\}$ as Fig.2.

Proposition 1. For the input places of $t$,

$$
M_{p_{i}}(\tau)=\operatorname{Mark}\left(p_{i}, \tau\right)+M_{t}(\tau) W\left(p_{i}, t\right) i=1,2, \ldots, n ;
$$

for the output places of $t$,

$$
M_{q_{i}}(\tau)=M_{q_{i}}(0)+M_{t}(\tau) W\left(t, q_{i}\right) i=1,2, \ldots, m .
$$

Proposition 2. Let $t \in T$, there exists $\Delta \tau>0$ satisfied

$$
M_{t}(\tau+\Delta \tau)=M_{t}(\tau)+\min \left\{V(t) \cdot \Delta \tau, M_{p_{i}}(\tau+\Delta \tau)-M_{t}(\tau) \cdot W\left(p_{i}, t\right), \quad i=1, \ldots, n\right\} .
$$

Proof. Assume that $\operatorname{Mark}\left(p_{i}, \tau\right)>0 \quad i=1,2, \ldots, n$. let

$$
0<\Delta \tau<\min _{i}\left\{\frac{\operatorname{Mark}\left(p_{i}, \tau\right)}{W\left(p_{i}, t\right) V(t)}\right\},
$$

then $\bar{V}(t, \tau)=V(t)$ holds in time interval $[\tau, \tau+\Delta \tau]$; and

$$
M_{p_{i}}(\tau+\Delta \tau)-M_{t}(\tau) \cdot W\left(p_{i}, t\right) \geq M_{p_{i}}(\tau)-M_{t}(\tau) \cdot W\left(p_{i}, t\right)>V(t) \cdot \Delta \tau,
$$

thus (3) satisfied.

Now consider the case that $\exists p \in{ }^{\circ} t \operatorname{Mark}(p, \tau)=0$ :

1) if there exists a $\Delta \tau>0$ such that $\min _{i}\left\{\operatorname{Mark}\left(p_{i}, \tau_{1}\right)\right\}=0$ holds for all $\tau \leq \tau_{1} \leq \tau+\Delta \tau$, then Definition 3 implies (3);

2) else there exists no $\Delta \tau>0$ such that $\min _{i}\left\{\operatorname{Mark}\left(p_{i}, \tau_{1}\right)\right\}=0$ holds for all $\tau \leq \tau_{1} \leq \tau+\Delta \tau$. As the limiting case of $\operatorname{Mark}\left(p_{i}, \tau\right)>0 \quad i=1,2, \ldots, n$, inequality (3) still holds in this case.

It is impossible to represent the equations in Proposition 2 as max-plus algebraic linear form, since there have some multiplication operations that are exponential operations under max-plus algebra.

\section{Dioid Representation of a Class of CEGs}

$M$ is a collection of functions, $\forall \lambda \in M \lambda: \mathrm{R} \rightarrow \mathrm{R}^{+}$, and $\lambda$ is monotone nondecreasing. A minimization operation $\oplus$ in $\bar{R}$ is defined by

$$
\forall r_{1}, r_{2} \in \bar{R} \quad r_{1} \oplus r_{2}=\min \left(r_{1}, r_{2}\right)
$$


Define the operation $\oplus$ in $M$ as point wise minimization

$$
\forall \lambda_{1}, \lambda_{2} \in M \quad \lambda_{1}(\tau) \oplus \lambda_{2}(\tau)=\min \left(\lambda_{1}(\tau), \lambda_{2}(\tau)\right)
$$

Obviously, $\forall \lambda \in M 0 \oplus \lambda(\tau)=\lambda(\tau)$ and $0 \in M$.

Proposition 3. $\langle M, \oplus\rangle$ is a monoid, the identity element is $0, \oplus$ is commutative and idempotent.

Proposition 4. $\forall\left\{\lambda_{i}\right\} \subseteq M \bigoplus_{i} \lambda_{i}(\tau) \in M$, namely $M$ is complete.

A partial-order $\leq$ is induced from $\oplus$ as follow:

$$
\forall \lambda_{1}, \lambda_{2} \in M \lambda_{1}(\tau) \leq \lambda_{2}(\tau) \Leftrightarrow \lambda_{1}(\tau) \oplus \lambda_{2}(\tau)=\lambda_{2}(\tau) .
$$

Definition 5. Mapping $f: M \rightarrow M$ is said to be lower-semicontinuous (1.s.c.) [3] if

$$
\forall\left\{\lambda_{i}\right\} \subseteq M \quad \bigoplus_{i} f\left(\lambda_{i}(\tau)\right)=f\left(\bigoplus_{i} \lambda_{i}(\tau)\right)
$$

Proposition 5. All the 1.s.c. mappings on $M$, denoted $F$, can be induced a dioid with two operations, $\oplus$ and $\otimes$, defined as follow:

$$
\begin{aligned}
\forall f_{1}, f_{2} \in F \forall \lambda \in M \quad & \left(f_{1} \oplus f_{2}\right)(\lambda)=f_{1}(\lambda) \oplus f_{2}(\lambda) \\
& \left(f_{1} \otimes f_{2}\right)(\lambda)=f_{1}\left(f_{2}(\lambda)\right) .
\end{aligned}
$$

According to Proposition 4 and Proposition 5, the following holds.

Corollary 1. F is Complete.

For briefness, $\otimes$ may be omitted.

Proposition 6. Let $k \in N$ and $k>0, x \in M^{k}$ be a variable vector, $v \in M^{k}$ be constant one, and $A \in F^{k \times k}$, the least solution of equations $x=A x \oplus v$ is $x=A^{*} v$, where $A^{*}=\bigoplus_{i=0}^{\infty} A^{i}[1]$.

Definition 6. $\forall \lambda \in M$

1) add mapping $\left[r^{+}\right], r \in[0,+\infty):\left[r^{+}\right] \lambda(\tau)=\lambda(\tau)+r$;

2) multiplication mapping $\left[r^{\times}\right], r \in R^{+}:\left[r^{\times}\right] \lambda(\tau)=r \cdot \lambda(\tau)$.

The symbol [] may be omitted if no ambiguity. Note that $0^{+}$and $1^{\times}$are both the identity element of $\langle M, \otimes\rangle$.

Let $F_{1}=\left\{\left[r^{+}\right] \mid r \in[0,+\infty)\right\}, F_{2}=\left\{\left[r^{\times}\right] \mid r \in R^{+}\right\}$, and

$$
F_{0}=\left\{\bigoplus_{i=1}^{m} \bigotimes_{j=1}^{n} f_{i j} \mid f_{i j} \in F_{1} \cup F_{2} \quad i, j, m, n \in N\right\} .
$$

Theorem 1. $F_{0} \in F$.

Proof. Because all the elements of $F_{1} \cup F_{2}$ is 1.s.c. . 
Given a class of CEGs satisfied

$$
\forall t \in T \max \left\{V\left(t_{i}\right) \frac{W\left(t_{i}, p_{i}\right)}{W\left(p_{i}, t\right)} \quad t_{i} \in{ }^{\circ}\left({ }^{\circ} t\right), p_{i}=t_{i}^{\circ}\right\} \leq V(t) .
$$

Theorem 2. Performances of the class of CEGs above mentioned could be described as linear algebraic equations.

Proof. Considering a transition $t_{k} \in T_{C}$, given that ${ }^{\circ}\left({ }^{\circ} t_{k}\right)=\left\{t_{k i} \mid t_{k i} \in{ }^{\circ}\left({ }^{\circ} t_{k}\right)\right\} \subseteq T_{C}$, as above assumed. Let $v_{k}(\tau)=V\left(t_{k}\right) \tau, p_{k i}=t_{k i}^{\circ}, m_{k i}=\frac{M_{p_{k i}}(0)}{W\left(p_{k i}, t_{k}\right)}, w_{k i}=\frac{W\left(t_{k i}, p_{k i}\right)}{W\left(p_{k i}, t_{k}\right)}$. The following equation holds.

$$
M_{t_{k}}(\tau)=\min \left\{V\left(t_{k}\right) \tau, \frac{M_{p_{k i}}(0)+M_{t_{k i}}(\tau) \cdot W\left(t_{k i}, p_{k i}\right)}{W\left(p_{k i}, t_{k}\right)}\right\}
$$

In fact, according to (12), on the one hand once a transition $t_{k i}$ satisfied $\operatorname{Mark}\left(t_{k i}, \tau_{0}\right)=0, \forall \tau \geq \tau_{0} \exists k_{i^{\prime}}$ s.t. $\operatorname{Mark}\left(t_{k i^{\prime}}, \tau\right)=0$; On the other hand, let $\tau_{0}=\min \left\{\tau \mid \neg \exists t_{k i} \operatorname{Mark}\left(t_{k i}, \tau\right)=0\right\}$, then $\tau_{0}=0$. (13) can be represented as follow:

$$
M_{t_{k}}(\tau)=\bigoplus_{k i}\left[m_{k i}{ }^{+}\right]\left[w_{k i}{ }^{\times}\right] M_{t_{k i}}(\tau) \bigoplus v_{k} .
$$

Let $\boldsymbol{x}=\left(t_{1}, t_{2}, \ldots, t_{|T|}\right)^{T}$ and $\boldsymbol{v}=\left(v_{1}, v_{2}, \ldots, v_{|T|}\right)^{T}$. (14) imply

$$
\boldsymbol{x}=\boldsymbol{A x} \oplus \boldsymbol{v}
$$

where $\boldsymbol{A}=\left(a_{i j}\right)$ is a mapping matrix, and $a_{i j}=\left[m_{i j}{ }^{+}\right]\left[w_{i j}{ }^{\times}\right]$.

Corollary 2. The least solution to (15) is $\boldsymbol{x}=\boldsymbol{A}^{*} \boldsymbol{v}$.

\section{Example of Optimal Control}

A manufacturing system represented by a CEG is shown in Fig. 5, where $V\left(t_{1}\right)=1, V\left(t_{2}\right)=1, V\left(t_{3}\right)=1, V\left(t_{4}\right)=1, M_{p_{1}}(0)=1, M_{p_{2}}(0)=0, M_{p_{3}}(0)=1$, $M_{p_{4}}(0)=1, M_{p_{5}}(0)=1$ and $M_{p_{6}}(0)=m$.

Using Theorem 2 , the following equations holds

$$
\boldsymbol{M}(\tau):=\left[\begin{array}{c}
M_{t_{1}}(\tau) \\
M_{t_{2}}(\tau) \\
M_{t_{3}}(\tau) \\
M_{t_{4}}(\tau)
\end{array}\right]=\left[\begin{array}{cccc}
\varpi & 1^{+} & \varpi & \varpi \\
1^{+} & \varpi & m^{+} & \varpi \\
\varpi & 1^{+} & \varpi & 1^{+} \\
\varpi & 1^{+} & \varpi & \varpi
\end{array}\right]\left[\begin{array}{c}
M_{t_{1}}(\tau) \\
M_{t_{2}}(\tau) \\
M_{t_{3}}(\tau) \\
M_{t_{4}}(\tau)
\end{array}\right] \oplus\left[\begin{array}{l}
\tau \\
\tau \\
\tau \\
\tau
\end{array}\right]:=\boldsymbol{A M}(\tau) \oplus \boldsymbol{v}
$$




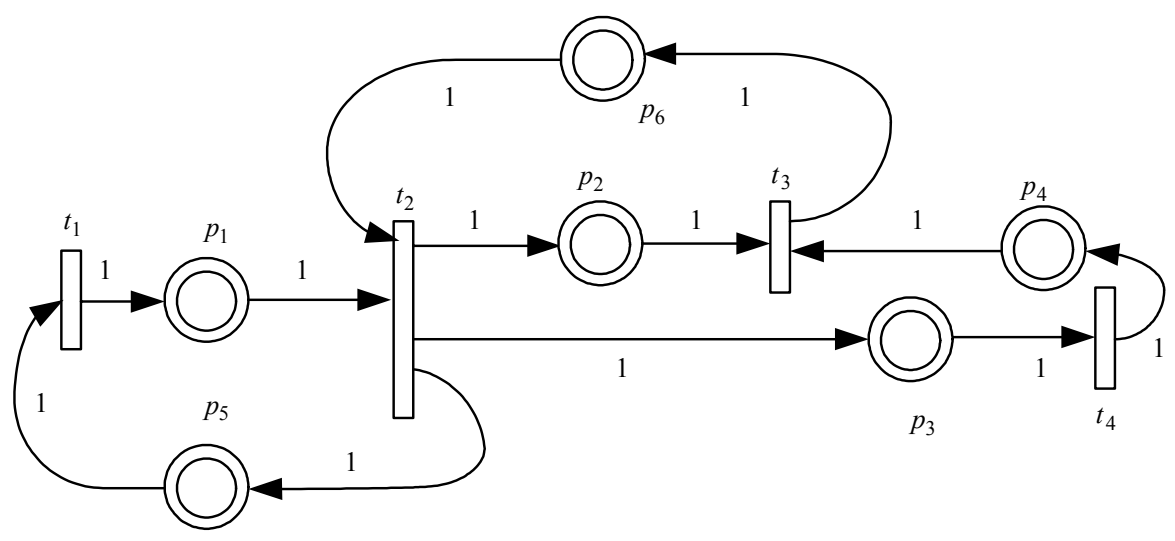

Fig. 3. Example of represented a CEG by dioid Equations

According Corollary 2, the least solution to (16) is

$$
A^{\bullet} v=\left[\begin{array}{cccc}
0^{+} & 1^{+} & (m+1)^{+} & (m+2)^{+} \\
1^{+} & 0^{+} & m^{+} & (m+1)^{+} \\
2^{+} & 1^{+} & 0^{+} & 1^{+} \\
2^{+} & 1^{+} & (m+1)^{+} & 0^{+}
\end{array}\right]\left[\begin{array}{l}
\tau \\
\tau \\
\tau \\
\tau
\end{array}\right]
$$

The solution indicate that $m$ are cannot affect the firing velocity of transition $t_{3}$, but that of $t_{1}, t_{2}$ and $t_{4}$.

\section{Conclusion}

In this note, a linear algebraic model $\langle F, \oplus, \otimes\rangle$ for performance evaluation of a class of Continuous event graph has been developed. And an interesting and useful result of the example gives us a novel approach to compute the minimum (optimal) initial tokens of places in a CEG by its algebraic model.

\section{Acknowledgement}

This work was supported by National Natural Science Foundation of China (60304018). 


\section{References}

1. Baccelli, F., Cohen, G., Olsder, G.J., Quadrat, J.P.: Synchronization and Linearity: An Algebra for Discrete Event Systems. Wiley, New York (1992)

2. Cohen, G., Gaubert, S., Quadrat, J.P.: Timed-events graphs with multipliers and homogeneous Min-plus systems. IEEE Trans. Automat.Contr. 43(1998) 1296-1302

3. Dai, H., Sun, Y.: An Algebraic Model for Performance Evaluation of Timed Event Multigraphs. IEEE Trans. Automat. Contr. 48(2003) 1227-1230

4. David, R, Alla, H.: Petri Nets Grafcet Tools for Modeling Discrete Event Systems. Hermes, Paris (1992).

5. David, R., Alla, H.: Continuous Petri Nets. European Workshop on Application and Theory of Petri Nets. Saragosse (1987)

6. David, R., Alla, H.: Autonomous and Timed Continuous Petri Nets. International Conference on Application and Theory of Petri Nets. Paris (1990) 367-386

7. David, R., Xie, X., Alla, H.: Properties of Continuous Models of Transfer Lineswith Unreliable machines and Finite Buffers. IMA Journal of Math. Applied in Business \& Industry. 6 (1990) 281-308

8. Alla, H., David, R.: Modeling of Production Systems by Continuous Petri Nets. 3rd International Conference on CAD/CAM, CARS \& FOF88. Detroit 3(1988)

9. Le Bail, J., Alla, H., David, R.: Hybrid Petri Net. European Control Conference. Grenoble (1991) 1472-1477

10. Le Bail, J., Alla, H., David, R.: Asymptotic Continuous Petri Nets: An efficient Approximation of Discrete event Systems. IEEE International Conference on Robotics and Automation. Nice (1992)

11. Zerhouni, N., Alla, H.: Asymptotic Continuous Petri Nets: An efficient Approximation of Discrete event Systems. IEEE International Conference on Robotics and Automation. Cincinnati. Cincinnati 2(1990) 1070-1075

12. Baccelli, F., Hong, D.: TCP is Max-Plus Linear and what it tells us on its throughput. INRIA Report. Paris (2000) 